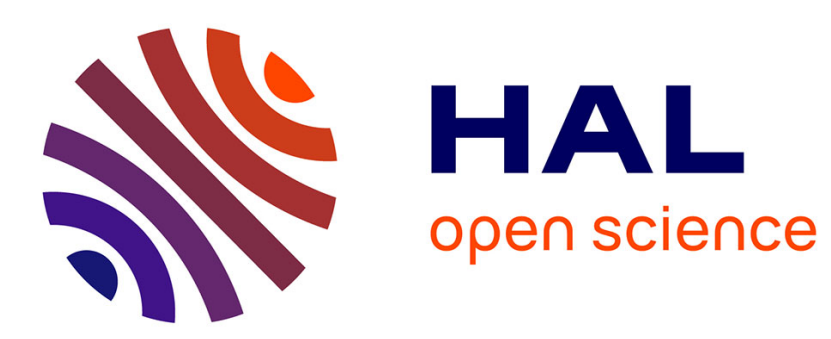

\title{
Les gestes rituels autour des papyrus-amulettes (Égypte, fin du IIe millénaire av. n. è.)
}

Sylvie Donnat

\section{To cite this version:}

Sylvie Donnat. Les gestes rituels autour des papyrus-amulettes (Égypte, fin du Ile millénaire av. n. è.). Archimède: archéologie et histoire ancienne, 2020, 7, pp.37-50. halshs-02893129

\section{HAL Id: halshs-02893129 \\ https://shs.hal.science/halshs-02893129}

Submitted on 8 Jul 2020

HAL is a multi-disciplinary open access archive for the deposit and dissemination of scientific research documents, whether they are published or not. The documents may come from teaching and research institutions in France or abroad, or from public or private research centers.
L'archive ouverte pluridisciplinaire HAL, est destinée au dépôt et à la diffusion de documents scientifiques de niveau recherche, publiés ou non, émanant des établissements d'enseignement et de recherche français ou étrangers, des laboratoires publics ou privés. 


\section{ARCHIMĖDE N N 7}

DOSSIER THÉMATIQUE :

GESTES RITUELS. DE LA TRACE À L'INTERPRÉTATION

1 Sylvie DONNAT, Jean-Marie HUSSER

Le rite et ses traces. Perspectives méthodologiques

6 Marie AUGIER

Corps et objets interdits dans les sanctuaires (monde grec, ve av. - III ${ }^{\mathrm{e}}$ ap. J.-C.)

23 Claire CAMBERLEIN

Offrir l'antique à la divinité. L'exemple de l'adyton du sanctuaire de Vryokastro sur l'île de Kythnos (Cyclades)

37 Sylvie DONNAT

Les gestes rituels autour des papyrus-amulettes (Égypte, fin du IIe millénaire av. n. è.)

51 Jean-Marie HUSSER

Entre rituel, mémorial et littérature de propagande. À propos de deux textes ougaritiques au genre mal défini

62 Anne JACQUEMIN

Rituels efficaces pour fonder ou déplacer un culte en Grèce ancienne

76 Françoise LAROCHE-TRAUNECKER

Les rituels de construction des temples égyptiens. Gestes représentés sur les parois et actes attestés par des fouilles à Karnak

96 Denis MONNERIE

Circulation des objets et élaboration des relations dans les cérémonies du nord de la Kanaky Nouvelle-Calédonie

\section{ACTUALITÉ DE LA RECHERCHE}

294 VARIA 


\title{
LES GESTES RITUELS AUTOUR DES PAPYRUS-AMULETTES (ÉGYPTE, FIN DU IIE MILLÉNAIRE AV. N. È.)
}

\author{
Sylvie DONNAT \\ Maîtresse de conférences en égyptologie \\ Université de Strasbourg \\ UMR 7044 Archimède \\ Chercheuse associée à l'UMR 8210 ANHIMA \\ sylvie.donnat@misha.fr
}

\section{RÉSUMÉ}

Les amulettes graphiques (texte et images) sur papyrus de l'époque ramesside et immédiatement post-ramesside (c. 1300-1000 av. n. è.) sont des objets rituels épigraphes dont l'étude nécessite la prise en compte, à côté de l'analyse philologique du texte et iconologique des images, de leur dimension matérielle. Elles constituent les vestiges de pratiques rituelles mobilisant l'écrit et le dessin, et portent des traces permettant de restituer une partie de la chaîne opératoire du rite. Les parallèles ethnographiques permettent par ailleurs de susciter des questionnements sur des points difficilement appréhendables en raison de la rareté des données textuelles et archéologiques : celui du désinvestissement des amulettes une fois que leur port n'était plus consi-

\section{MotS-CLÉS}

Amulettes textuelles, écriture rituelle,

chaîne opératoire, papyrus Deir el-Médîna 36, papyrus Deir el-Médîna 44 . anthropologique.
Ramessid textual amulets on papyrus are epigraphic artefacts made during a ritual procedure. They show traces of an operational chain. Ethnographical parallels are useful to raise questions about the final manipulation and deposition of the amulet, once it is not worn anymore. With an anthropological oriented questioning, the pairing of the philological and archaeological tools is essential to reconstruct the ritual gestures and to interpret them.
KEYWORDS

Textual amulets, writing as ritual practise, operative chain, papyrus Deir el-Medina 36 papyrus Deir el-Medina 44. 
Un texte est, dans sa dimension écrite, un objet matériel. C'est une évidence qu'il n'est pas toujours inutile de rappeler. L'écriture, en effet, est « un objet matériel qui résulte de l'acte d'écrire et se conserve dans le temps et l'espace : c'est un objet physique qu'on peut étudier comme tel (archéologie du livre) ; le tracé des caractères, des lettres, est aussi un objet matériel dont l'étude nous replace dans le temps et l'espace de l'acte d'écrire et nous renseigne sur le scripteur et son époque [1]. " Dans certains contextes, l'écrit peut plus spécifiquement constituer une trace matérielle de rite. C'est le cas des textes relevant de la catégorie des " écritures rituelles [2] ". J'entends ici la notion comme se référant à des écrits dont l'acte graphique (l'écriture et le dessin) peut avoir fait l'objet d'une ritualisation et qui ont été mobilisés dans la performance rituelle. En contexte égyptien, et en s'en tenant à la tachygraphie manuscrite dite " hiératique [3] ", on peut, sans prétendre à l'exhaustivité [4], inclure dans cette catégorie :

[1] SiRAT 1990, p. 3. La partie finale de cette citation est reprise dans RAGAZZOLI 2019, p. 39, avec un commentaire sur l'archéologie du manuscrit.

[2] Sur cette notion : KOCH \& BATSCH 2010. Cf. DONNAT 2019b, avec références.

[3] Écriture cursive manuscrite se présentant comme une simplification des signes hiéroglyphiques, et autorisant les ligatures entre les signes, en usage dès le début du III millénaire av. n. è. C'est l'écriture usuelle de la pratique scribale quotidienne (administration, communication épistolaire, belles lettres, enregistrements de savoirs...), jusqu'à ce que l'invention de la tachygraphie démotique au $\mathrm{VII}^{\mathrm{e}}$ siècle av. $\mathrm{n}$. è. ne vienne redéfinir ses usages. Sur les usages du hiératique, en particulier au Nouvel Empire, voir RAGAZZOLI 2019, p. 25-26.

[4] Cf. DonNAT 2015. Sur l'écriture et la religion égyptienne de manière générale : voir Goody 1986, p. 38-48 ; CARDONA 1994, chapitre 5, p. 147-184. AsSMANN 2000 (pour l'écrit dans la religion funéraire), spécialement p. 29-37.
- les figurines d'ennemi et céramiques inscrites utilisées dans les rites d'exécration [5]

- les lettres aux morts, utilisées dans la communication vivant-mort [6]

- les lettres aux dieux, utilisées dans la communication vivant-divinité [7]

- les questions aux oracles, utilisées dans la consultation d'une divinité oraculaire [8]

- les papyrus-amulettes utilisés pour la protection du corps d'un individu vivant [9], ou ceux utilisés pour la protection du corps d'un défunt [10].

Il y aurait bien évidemment beaucoup à dire sur ce que dévoilent ces pratiques de l'histoire de la culture de l'écrit égyptienne et de ses rapports avec la religion [11]. Mais tel n'est pas le propos de la présente contribution, qui aborde la question sous un angle méthodologique : d'une part, ces artefacts écrits constituent les témoignages de l'exécution d'un rite qui nécessitait la présence

[5] JAMBON 2010.

[6] Donnat Beauquier 2014 ; Donnat 2014a ; FRANKFURTER 2018 ; DONNAT 2019a.

[7] LEFEVRE 2017 ; FRANKFURTER 2018.

[8] Valbelle \& Husson 2001 ; Grandet 2003, p. 9.

[9] Les « amulettes textuelles », objets de la présente contribution (voir DieLEMAN 2015 ; DONNAT 2016a ; DiELEMAN \& Fischer-ELFERT 2017 ; KOENIG 2004), mais aussi les oracular amuletic decrees de la première moitié du Ier millénaire av. n. è. (XXIe-XXII e dynasties) : EDWARDS 1960 ; LUCARELLI 2009.

[10] Sur les Totenbuchamulett attestées à partir de la XXI dynastie et pendant le Ier millénaire av. n. è., voir ILLÈs 2006 ; Demichelis 2000 ; Albert \& GABOlDE 2013 : FisCHER-ELFERT 2015, cat. 1, p. 77-81 et cat. 10 , p. 152-156 ; RÉGEN 2017, p. 99 ; RÉGE, à paraître.

[11] Cf. de manière générale Goody 1986, p. 1-37 ; GoodY 2007, p. 131-175. 
d'un élément graphique [12] ; ils portent, d'autre part, des traces qui sont autant d'indices de la chaîne opératoire de leur fabrication et des manipulations rituelles dont ils ont fait l'objet.

À cet égard, les amulettes inscrites sur papyrus d'une formule (incantation, invocation, conjuration...) en hiératique, pour des individus vivants, constituent un objet d'étude intéressant. Pour tenter de restituer la pratique (depuis la fabrication de l'amulette, jusqu'à son retrait, en passant par son installation sur le corps du bénéficiaire), une enquête convoquant les analyses et approches du philologue, de l'iconologue, du papyrologue, de l'archéologue et de l'anthropologue est en effet nécessaire. Dans cette contribution, je propose de donner un aperçu des différentes pistes méthodologiques qui peuvent être mises en œuvre et combinées pour tirer le maximum de l'objet papyrus-amulette. Bien que ces objets soient attestés à la fin du $\|^{\mathrm{e}}$ millénaire et pendant le $\mathrm{I}^{\mathrm{er}}$ millénaire [13], l'étude se focalise sur le corpus le plus ancien, celui daté de l'époque ramesside ( $\mathrm{XI} \mathrm{X}^{\mathrm{e}}-\mathrm{X} \mathrm{X}^{\mathrm{e}}$ dynasties) en incluant la période immédiatement postérieure (environ entre 1300 et 1000 av. n. è.) [14]. Malgré la pertinence d'une enquête qui opèrerait une comparaison entre diverses catégories de " papyrus-amulettes " en hiératique, dans cette étude, je laisse volontairement de côté les Totenbuchamulett et les Oracular Amuletic Decrees $(O A D)$ [15]. Ils ne font en effet leur apparition qu'à partir du début de la XXI ${ }^{\mathrm{e}}$ dynastie (1069945 av. n. è.), et surtout ils relèvent soit d'un champ d'application différent (le funéraire pour les Totenbuchamulett [16], avec les spécificités rituelles afférentes), soit d'un registre discursif et d'un référent rituel distincts (consignation d'une parole censément [17] oraculaire - stéréotypée - pour les $O A D$ ).

\section{DESCRIPTION GÉNÉRALE DU CORPUS DES AMULETTES TEXTUELLES RAMESSIDES}

Les papyrus-amulettes en hiératique sont archéologiquement attestés en Égypte ancienne environ à partir de 1300 av. n. è., c'est-à-dire pendant l'époque ramesside [18]. Ils sont datés sur la base de critères paléographiques, philologiques et archéologiques. À cette période, les amulettes se présentent généralement sous la forme d'un morceau quadrangulaire de papyrus, de dimensions variables (largeur standard indicative environ $21 \mathrm{~cm}$, hauteur variable environ entre 8 et $22 \mathrm{~cm}$ ) [19], inscrit à l'encre noire (avec occasionnellement des éléments en rouge [20]) d'une formule écrite dans la cursive dite " hiératique " et de dessins tracés. Lorsqu'ils sont mis au jour, ils se présentent dans leur état final, c'est-à-dire sous la forme de petits paquets de papyrus, pliés et fermés d'un cordon de lin, d'environ 2 sur $4 \mathrm{~cm}$ (fig. 1). Ces objets, appelés médjat ( $m \underline{d} 3 . t-$ « rouleau,

Figure 1

Papyrus-amulette plié (PDM 44). D’après Koenig 1999, p. 259. (C IFAO.

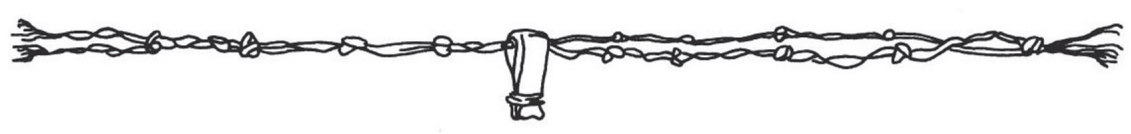

[12] La ritualisation potentielle de l'acte graphique luimême est une question souvent très difficile à trancher. Voir JAMBON 2010, § 56-70 ; aussi les remarques conclusives dans la présente contribution.

[13] Voir l'inventaire de DiELEMAN 2015, p. 49-51.

[14] Une recherche est actuellement en cours portant spécifiquement sur un sous-dossier d'amulettes de la fin du II ${ }^{\mathrm{e}}$ millénaire av. n. è., dirigées contre l'inflammation séref. Voir DONNAT 2016b.

[15] Voir supra, n. 9.

[16] Voir supra, n. 10.

[17] Voir les remarques de WINAND 2003, p. 612, sur les décrets oraculaires avec et sans consultation préalable.
[18] Pour l'hypothèse de l'existence plus ancienne (dès le Moyen Empire) de pratiques graphiques comparables, voir KoEnIG 1979, p. 119. Voir aussi la position de DiELEMAN 2015, p. 23.

[19] La largeur standard du feuillet découpé correspond probablement à la hauteur d'un rouleau de papyrus de $21 \mathrm{~cm}$ - c'est-à-dire un rouleau représentant la moitié du rouleau grand format de $42-44 \mathrm{~cm}$ de l'époque ramesside. Voir Koenig 1982, p. 29 et n. 4 ; DiELEMAN \& FisCHERELFERT 2017, p. 245-246 et n. 11 . Les dimensions des amulettes sont toutefois variables, particulièrement selon les époques. Pour une typo-chronologie des formats, DiELEMAN 2015, p. 49-52.

[20] Amulette P. hiérat. 69 de la BNU de Strasbourg : DONNAT 2016b. 
livre " [21]) en égyptien ancien, sont désignés dans la littérature scientifique sous diverses expressions : " billets magiques ", "phylactères ", " papyrus amulettiques ", " amulettes iatromagiques [22] " ou " amulettes textuelles" (textual amulets [23]). Cette dernière appellation, particulièrement utile dans le cadre d'une approche comparatiste [24], ne rend toutefois peut-être pas suffisamment compte de l'importance des images dans les objets pharaoniques [25]. Un inventaire exhaustif de ces amulettes reste encore à faire, mais, pour l'époque ramesside, un recensement provisoire en signale vingt-quatre [26]. Sur cet ensemble, comme on le verra, une proportion relativement réduite de papyrus possède une provenance archéologique, ce qui n'est pas sans poser un certain nombre de problèmes.

\section{LES SOURCES TEXTUELLES}

Les sources textuelles documentant la pratique sont en revanche plus nombreuses. Comme on peut s'y attendre, elles laissent toutefois nombre de questions en suspens. On peut classer ces sources en deux catégories. Il y a d'une part celles qui peuvent être qualifiées d'externes : textes prescriptifs présents dans les manuels rituels (véritables " billets modèles [27]" ou formules présentant un rite graphique comparable [28]), plus exceptionnellement évocation dans un texte littéraire [29]. On trouve, d'autre part, des sources textuelles qui sont internes au corpus: les textes écrits sur les amulettes elles-mêmes. Ces dernières présentent en effet, sur leur face principale

\section{[21] FisCheR-ElFERT 2015, p. 135.}

\section{[22] COULON 2018, p. 117.}

[23] Pour un aperçu de ces appellations avec état de la question et bibliographie, voir DONNAT 2016a, et renvoi à DIELEMAN 2015, pour la désignation textual amulets.

[24] Sur l'emploi de la désignation textual amulets en dehors du matériel égyptologique, voir par exemple VentURA et al. 2014, avec bibliographie. Cf. aussi SKEMER 2014.

[25] ESCHWEILER 1994, p. 59-68, pour les images dessinées dans les rites apotropaïques. Cf. KoENIG 2015, pour des amulettes sur papyrus ne comportant que des images, et AZZAM 2017 pour une « amulette textuelle » « abrégée » (P. Leyde I 353 = RMO AMS 59e), centrée essentiellement sur les images, avec, pour seul texte, le titre et le mode d'emploi.

[26] Dieleman \& Fischer-ELFERT 2017, p. 257, n. 74 (24, sans la liste des papyrus). Cf. Dieleman 2015, p. 49 (12 items listés).

[27] GARDINER 1935, vol. 1, p. 65, vol. 2 pl. 38-38A ; voir aussi la traduction et commentaire, sur le site cité infra, n. 33. (recto), le texte de l'incantation [30] qui, si on en croit les notices prescriptives, devait être prononcé lors du rituel d'installation de l'amulette. Souvent ce texte est accompagné de son apparat éditorial (titre et/ou notice prescriptive). Celui-ci n'avait pas vocation à être verbalisé lors du rite. II relève strictement d'un usage scribal [31], mais il jouait néanmoins vraisemblablement un rôle dans la construction de l'efficacité de l'amulette : probablement, la mise en page du texte, en reprenant celle des manuels rituels, convoquait graphiquement un discours d'autorité, un savoir de lettré hautement valorisé [32].

La notice prescriptive occasionnellement incluse dans le texte des amulettes est, dans sa structure, tout à fait identique à celle des billets modèles.

Billet modèle du P. Chester Beatty VII, verso 7, notice prescriptive complète :

"Qu'on dise cette formule, ces tracés (étant) sur une feuille de papyrus [neuve, placée] au cou de l'homme " ( $d d=t w r(3) p n, n n n(y)$ sš.w hr $d m^{\prime}$ [n-m3w d.w r] hh $n(y) s$ [33]).

Amulette P. hiérat. 69 de la BNU de Strasbourg [34], rubrique prescriptive complète :

Dire ces paroles sur ces di[eux ?] (6) (du ?) rouleau (?), fait(s) par tracé sur [une feuille de papyrus neuve $\mathrm{n}$ ] puis placé au cou de I'homme. 》 (d dd-mdw hr $n n n(y) n[\underline{t}$ r.w] $m \underline{d}$ 3.t. ir irw $m$ sš hr [d

Amulette Papyrus British Museum EA 10732 [35], notice prescriptive abrégée :

«Dire ces paroles sur ces dieux » $(\underline{d} d m d w h r$ $n n n(y) n \underline{t}$ r.w)

[28] Par exemple, la prescription de placer une pièce de lin inscrite d'une formule $(r(3))$, sur le corps d'une femme malade, dans le Papyrus médical de Londres daté de la XVIII ${ }^{\mathrm{e}}$ dynastie (British Museum P. EA 10059, IX, 8-9), LEITZ 1999, p. 68.

[29] Voir infra, page suivante.

[30] Une exception notable déjà mentionnée ci-dessus en note 25 : le P. Leyde I 353 (RMO AMS 59e) ne présente que le paratexte (titre de la formule et mode d'emploi), et non I'incantation. Sur cette amulette, AzZAM 2017 ; FISCHER-ELFERT, à paraître.

[31] Cf. le commentaire dans DonNAT 2016b.

[32] DONNAT 2016b.

[33] Sur cette formule, GARDINER 1935, p. 65 ; la traduction de ESCHWEILER 1994, p. 37-38 et fig. 9 (« der im Schrift ist ») est à reconsidérer. Voir FISCHER-ELFERT 1998 , p. $109-110$; L. Popko, site Wissenschaft im alten Ägypten, http://sae.saw-leipzig.de/detail/dokument/ papyrus-chester-beatty-vii/ (dernière consultation 11/4/2019) ; DonNAT, 2020, à paraître.

[34] Édition DONNAT 2016b. Voir aussi FISCHERELFERT, à paraître.

[35] DONNAT, 2020, à paraître. 
Comme on peut le noter, l'acte rituel particulièrement mis en avant est, comme dans d'autres rites [36], celui d'une énonciation orale ( $d d$, « dire ») sur le dispositif graphique [37]. Les notices prescriptives insistent sur trois actes rituels, exprimés par trois verbes d'action [38] : $d d$ (" dire ») - iri (" façonner » l'amulette), éventuellement sš « tracer » dans le cadre de prescription graphique, - rdi (« placer, installer " l'amulette). Ce séquençage tripartite de l'action rituelle est fréquent dans les manuels de rituels apotropaïques; on le retrouve spécifiquement dans la prescription d'une amulette-médjat (amulette-papyrus) dans un recueil, pour la protection de la femme et de l'enfant, daté de l'époque ptolémaïque.

P. Brooklyn 42.218.2 x+ VII,17 [39], rubrique prescriptive (époque ptolémaïque) :

« Dire les paroles sur ces dieux qui sont tracés (ci-contre) ; façonner comme amulette-médjat, et placer au cou de l'enfant » ( $d d-m d w h r n n n(y)$

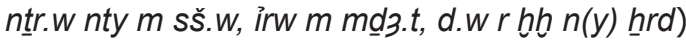

Il est bien clair que ces notices prescriptives ne décrivent pas l'entièreté de la procédure de fabrication de l'amulette (aucune mention, par exemple, du pliage du papyrus), mais rendent compte des actions probablement les plus signifiantes du point de vue de la logique rituelle : un acte verbal ( $\underline{d} d)$ est mis en avant, et deux actes non verbaux (façonnage et installation de l'amulette) [40].

Les sources littéraires extérieures, quant à elles, ne donnent pas plus de détails sur le processus de fabrication. Le cycle démotique de Setne, en particulier le conte de Setne 1 connu par un manuscrit daté de l'époque ptolémaïque, met bien en scène de façon très vivante des pratiques rituelles mobilisant l'écrit [41], mais les modalités présentées ne sont pas toujours identiques à celle des papyrus amulettiques. À ce jour, la seule œuvre littéraire ramesside connue pour évoquer directement ces objets est la Lettre satirique $d^{\prime}$ Hori. Le nombre des copies (complète et partielles) qui sont arrivées jusqu'à nous indique la grande diffusion de ce texte dans la culture scribale d'époque ramesside [42]. Comme le résume $\mathrm{Ch}$. Ragazzoli, cette composition, de nature didactique, a pour thématique le métier de scribe : le scribe Hori écrit une lettre à son supérieur, le scribe royal et militaire Aménémopé, à travers laquelle il met en cause les compétences professionnelles de ce dernier [43]. Le contexte polémique du passage qui nous intéresse est particulièrement clair, en dépit des difficultés de compréhension de certains termes. Hori reproche à Aménémopé de lui avoir envoyé une lettre mal tournée :

« Si tu avais su avant que ce n'est pas bon, alors tu ne l'aurais pas fait apporter (la lettre), en disant : "Les textes-bga passent leur temps attachés (?) à mes doigts, comme les rouleaux ( $m \underline{d} 3 . t)$ contre les maux au cou de celui qui souffre [44]. " (bsy=k $r h . t w h r-h$. $. t r-d d$ bn $n f r, t m=k$ d.t in.tw=s $r-d d$ : $n 3$ bg3w

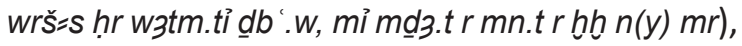

Selon Hori, le scribe incriminé aurait mieux fait de garder par devers lui sa lettre, comme un malade garde tout contre lui, de façon prolongée, les amulettes sur papyrus. Même s'il livre peu de détails sur la pratique rituelle, ce passage est significatif car il insiste sur une caractéristique essentielle de ces talismans : leur installation prolongée sur le corps du patient. Il témoigne en outre, non seulement, de ce que la pratique était suffisamment familière aux scribes pour pouvoir être citée comme contrepoint
[36] Sur le rite oral, GARDiner 1915, § 7.1, p. 264266. Sur l'importance de la parole rituelle, voir encore COULON 2010, p. 288 ; WEISS 2014.

[37] Sur le sens de « ces dieux » $(n n n(y) n t r . w)$ en ce contexte (comme désignation des vignettes), voir FISCHER-ELFERT 2015, p. 150, et n. 39 (renvoyant toutefois à la traduction d'une inscription de la tombe de Nefermaât à I'Ancien Empire par W. Spiegelberg, remise en cause par OSING 1984, p. 283-284; cf. le commentaire de StAUder 2018, p. 255). Voir encore Koenig 1981, p. 37-38.

[38] Pour une attention particulière portée aux actions et gestes rituels évoqués dans les textes égyptiens, voir RITNER 1994. Pour l'analyse des verbes d'action dans la description d'un rite sacrificiel grec : JAILLARD 2009, spécialement p. 66 ; voir aussi CARASTRO 2009, p. 272283, pour l'analyse de verbes du texte d'une lamelle (katadesmos) grecque.
[39] Germeur 2013, p. 20.

[40] Sur l'imbrication du verbal et du non verbal dans le rite, voir les références à Claude Lévi-Strauss dans L'homme nu (1971) et à Denis Monnerie citées dans DONNAT 2014b, p. 3, n. 13.

[41] Agut-Labordère \& Chauveau 2011, en particulier p. 26 (copie du Livre de Thot sur un papyrus neuf brûlé et dissout dans de l'eau avant ingestion) et p. 29 (suspension du papyrus de Thot au corps du héros au moyen d'une bande de lin).

[42] Mathieu 2002, p. 122 et tableau p. 134 ; RAGAZZOLI 2019, p. 190.

[43] RAGAZZOLI 2019, p. 190-193.

[44] Ce passage a été signalé par VERNUS 2010-2011, p. 66, n. 154 (Papyrus Anastasi I, 7, 2-4). Cf. Wente 1990, p. 102. 
dans une argumentation, mais aussi, me semblet-il, de ce qu'il pouvait exister un regard critique ou sceptique latent.

Comme on le voit, les sources textuelles disponibles, aussi riches soient-elles, laissent nombre de questions relatives à la séquence des gestes rituels sans réponse. En I'absence de description complète du rite, il faut bien évidemment se tourner vers I'examen matériel des artefacts eux-mêmes.

\section{TRACES DIRECTES DE LA CHAÎNE OPÉRATOIRE}

Cinq types d'observations, qui relèvent de la philologie matérielle [45] et de l'examen archéologique, permettent de proposer une restitution partielle de la chaîne opératoire de constitution de l'objet :

- l'examen de l'état final des amulettes (état de découverte),

- les traces de pliures (pour les amulettes qui ont été ouvertes lors de leur entrée dans les collections muséales),

- la présence de dessin(s) au verso,

- les empreintes d'encre,

- la rapidité de l'écriture,

- et la fréquence, comme la qualité, des recharges d'encre.

\section{ÉTAT FINAL}

Dans leur état final (de découverte), et avant leur ouverture, les amulettes se présentent, on l'a dit, sous la forme de petits paquets de quelques centimètres pliés plats, fermés par un cordon de lin [46] (parfois avec un sceau en argile [47]) et associés à un collier à nœuds. Le texte et les images étaient donc invisibles au regard [48]. Le dispositif qui permettait de suspendre l'amulette n'est pas évoqué explicitement dans les rubriques prescriptives, même s'il est bien connu dans les prescriptions d'autres types d'amulettes. Par exemple, dans la formule $\mathrm{M}$ du Papyrus Berlin 3027 (Mutter und Kind) daté du début de la XVIII dynastie (vers 1550-1450 av. n. è.), les os d'une souris cuite sont à suspendre au cou de l'enfant au moyen d'une bande de lin fin à laquelle sept nœuds sont faits ( $r$ diw qs.w $r$ hh $h=f m$ stp $n(y)$ h. 3 ty, irs=tw 7 ts.wt) [49]. Le recueil du papyrus Leyde I 346 (= AMS 23a) recommande de fabriquer une amulette faite au moyen d'une pièce de lin sur laquelle est dessinée une série de douze divinités, pièce de lin ensuite façonnée au moyen de douze nœuds [50]. Dans le cas de l'amulette PDM 36, mise au jour par Bernard Bruyère dans le village de Deir al-Médîna, l'élément permettant la suspension est une pièce de lin, ellemême décorée de dessins apotropaïques et façonnée au moyen de nœuds [51].

\section{TRACES DU PLIAGE ET TRACES D'ENCRE MIROIR}

Lorsque l'on travaille sur une amulette ouverte et mise sous verre très anciennement (comme par exemple le papyrus hiérat. 69 de la BNU de Strasbourg), les traces de cet état final originel sont clairement visibles sous la forme de lacunes filiformes indiquant l'emplacement des plis. Très souvent, une lacune centrale plus importante que les autres signale l'endroit où l'épaisseur du pliage était la plus importante et où l'élasticité du papyrus a été le plus mise à mal. L'image, fréquemment dessinée au verso des billets, selon toute vraisemblance à la fin du processus, est aussi une indication du format final [52]. Les techniques de pliage des papyrus égyptiens ont été remarquablement étudiées par Myriam Krutzsch [53]. Ces techniques de pliage (par opposition aux techniques consistant à rouler un papyrus) concernent spécifiquement les amulettes ramessides, les lettres, et les petits documents [54]. Généralement, le pliage des amulettes ramessides s'opère d'abord par une série de plis (plats) successifs horizontaux (dans un seul sens ou deux, plis appelés Innere Faltung). La bande ainsi obtenue est ensuite pliée verticalement (plis appelés $\ddot{A} u ß e r e$ Faltung), de sorte que ces deux extrémités se trouvent à l'intérieur du petit paquet formé (fig. 1) [55]. Cette technique correspond au type Faltpäckchen III défini
[45] Voir RAGAZZOLI 2017, p. 95-96.

[46] Fischer-ELFERT \& DielemAn 2017, p. 246 et fig. 5.

[47] Fischer-ELFERT 2015, cat. 7, p. 132.

[48] Sur le pouvoir attribué à ce dispositif graphique caché, voir QUACK 2014, p. 38-39.

[49] YAMAZAKI 2003, p. 30-31. Sur les nœuds, WENDRICH 2006, spécialement p. 251-254.

[50] BOMMAS 1999, p. 252.
[51] SAUNERON 1970.

[52] DONNAT 2016b.

[53] Pour une typologie des pliages selon les types de textes, voir KRUTZSCH 2006. Pour l'analyse matérielle des textes magiques, voir spécifiquement KRUTZSCH 2015.

[54] KRUTZSCH 2015, p. 21 ; Dieleman \& FischeRELFERT 2017, p. 248. Cf. KRUTZSCH 2016, p. 62.

[55] KRUTZSCH 2015, p. 22. 
par M. Krutzsch. Des exceptions existent toutefois dans le corpus ramesside : I'amulette Berlin 15749, qui présentait encore, lors de son ouverture, le petit cachet d'argile scellant le document, a ainsi été pliée selon la technique Faltpäckchen II, c'est-à-dire avec une des deux extrémités du papyrus sur l'extérieur du paquet [56].

En dehors des traces de pliages, la surface interne des papyrus présente régulièrement des traces d'encre, qui ne sont que très exceptionnellement des traces de palimpseste [57] (la procédure rituelle interdit en théorie le recours à des papyrus de réutilisation [58]), mais plutôt des traces d'écriture spéculaire. De telles empreintes sont par exemple observables sur le papyrus hiérat. 69 de la BNU de Strasbourg ou sur le papyrus New York MMA 26.3.225. Elles peuvent permettre de restituer l'ordre du pliage avec un certain degré de certitude [59]. L'observation d'encre miroir est aussi fréquemment faite pour les amulettes iatromagiques en grec ; des expériences de fabrication de répliques tendraient à montrer la rapidité d'écriture et de pliage nécessaire pour obtenir de telles traces [60]. Ces éléments suggèrent donc que certains billets ont pu être pliés rapidement, avant que l'encre des signes concernés n'ait eu le temps de sécher entièrement [61]. La rapidité de la réalisation est en tout cas confirmée par la forme de l'écriture hiératique, généralement très nerveuse et peu calligraphique [62].

La prudence s'impose toutefois dans les conclusions qui peuvent être tirées de l'observation de certaines migrations d'encre, comme l'illustre le cas de l'amulette PDM 44 [63]. Le papyrus $(23 \mathrm{~cm}$ de hauteur sur 22,8 cm de largeur [64]) a été retrouvé
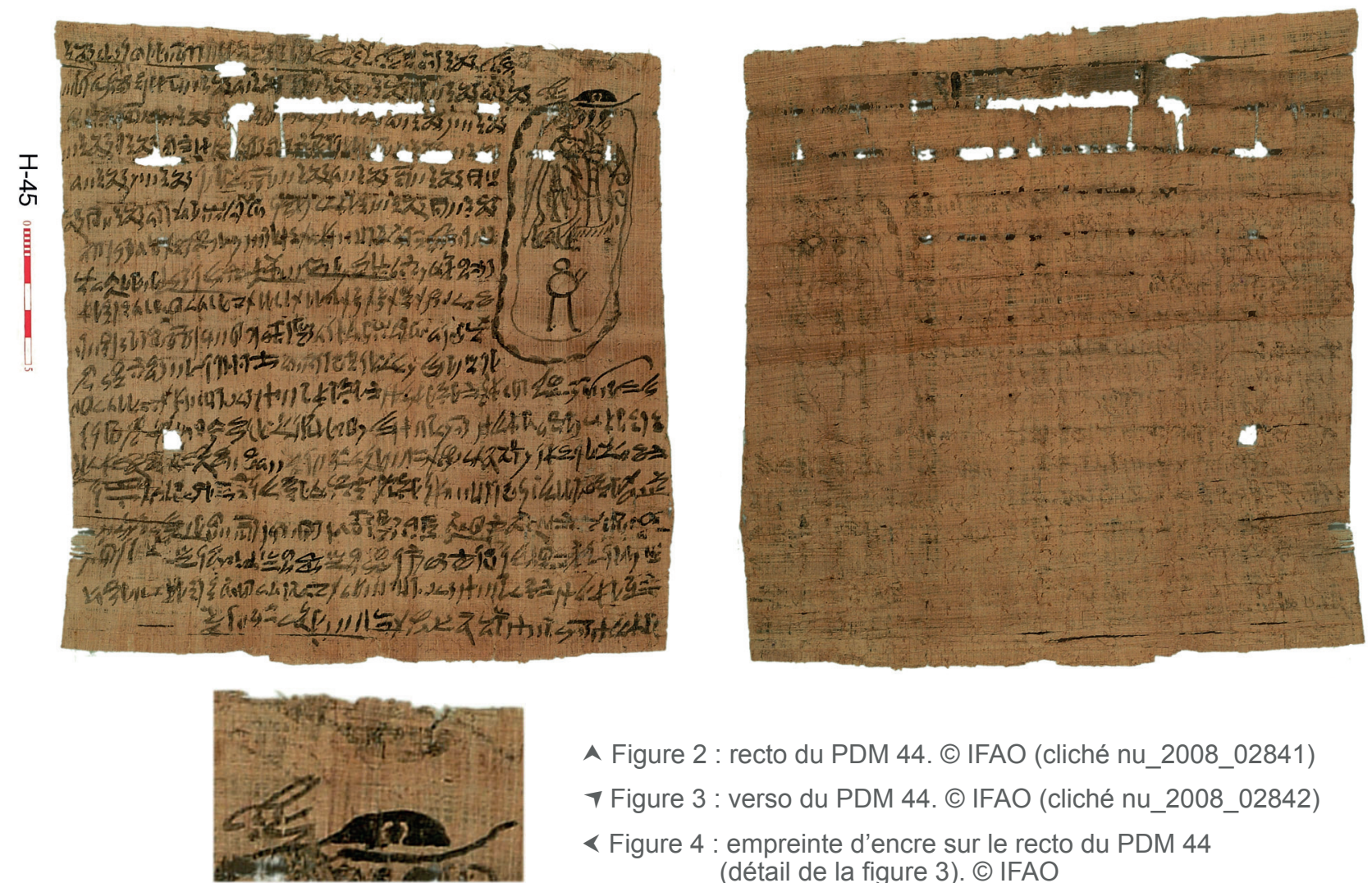

A Figure 2 : recto du PDM 44. ( IFAO (cliché nu_2008_02841)

$\checkmark$ Figure 3 : verso du PDM 44. (C IFAO (cliché nu_2008_02842)

< Figure 4 : empreinte d'encre sur le recto du PDM 44 (détail de la figure 3). (C IFAO

[56] KRUTZSCH 2015, p. 23 et schéma p. 31.

[57] Par exemple : I'amulette British Museum EA 10731 attribuée au scribe Qéniherkhépéchef (EDWARDS 1968, p. 155) ; I'oracular amuletic decree, Hanovre 1976.60c (FISCHER-ELFERT 2015, p. 202, cat. 19).

[58] FischeR-ELFERT 2015, p. 202, citant la notice prescriptive de l'amulette PDM 36 (SAUNERON 1970). Voir encore les textes cités, supra.

[59] Dieleman \& Fischer-Elfert 2017, p. 246, § 1.4. [60] HARO SANCHEZ 2012.

[61] Donnat 2016. Dieleman \& Fischer-Elfert 2017, p. 246.
[62] SAUNERON 1970, p. 7-8; Koenig 1999, p. 260 ; QUACK 2014, p. 37, qui remarque le peu de soin calligraphique, ce qui correspond au fait que les textes n'avaient effectivement pas vocation à être lus.

[63] Édition : Koenig 1999. Ce cas a été évoqué dans le cadre d'une communication faite lors de la journée d'étude « Intertextualité et intericonicité dans l'Égypte ancienne », PSL-EPHE, EA 4519, le 17 novembre 2018 : je remercie les participants pour leurs remarques qui m'ont conduite à reconsidérer ce document.

[64] Koenig 1999, p. 259, n. 2, citant le journal de fouilles de $B$. Bruyère. 
dans la tombe P 1444 de la colline de Gournet Mourraï qui jouxte, à l'est, le village de Deir al-Médîna. II se présentait sous la forme d'un « paquet ficelé par un brin de fil de lin ", traversé par un élément de suspension fait « de ficelle et de toile de $55 \mathrm{~cm}$ de longueur avec 9 nœuds à droite et 4 nœuds à gauche [65]» (fig. 1). D’après Bernard Bruyère, le paquet de papyrus a été ouvert le soir de la trouvaille. Selon sa description, il avait été plié « en commençant par le bas 17 fois sur lui-même, puis en sa largeur 2 fois de chaque côté du milieu ». Des traces de certains de ces plis (en particulier dans la partie supérieure du papyrus) sont bien visibles sur la photographie (fig. 2 et $\mathbf{3}$ ), sous la forme de séries de petites lacunes alignées. Une fois le papyrus déplié, sa face principale (le recto, fig. 2) présente une inscription de 19 lignes de hiératique et, en haut à droite, une vignette (ouroboros encerclant une figure de Thouéris, un personnage anthropomorphe tenant un crocodile par la gueule et une figure composite appelée « bonhomme têtard [66] » par Y. Koenig). Une empreinte d'encre est clairement visible sur la bordure supérieure droite : la tête de l'ouroboros, entièrement peinte en noire, est imprimée, en miroir, au-dessus, sur le bord supérieur (fig. 4). Cette marque indique qu'un pli a toutefois été effectué depuis le haut du papyrus. De cette façon, les extrémités supérieure et inférieure de la feuille se sont retrouvées à l'intérieur du pliage. Le verso, quant à lui, est recouvert, sur environ les $3 / 4$ de sa surface, d'empreintes d'encre (fig. 3). Sur la gauche, on voit très bien en particulier la migration de l'encre de l'ouroboros de la vignette, avec un décalage notable de plusieurs centimètres vers le bas. Ce décalage ne peut être obtenu que si, effectivement comme l'indique la description de Bruyère, l'essentiel du pliage a été réalisé en commençant par le bas du papyrus. Les empreintes d'encre sur le recto et le verso du papyrus et les traces de plis donnent ainsi des indications sur la chaîne opératoire probable du pliage de l'amulette PDM 44 . Elles complètent la description de $B$. Bruyère, bien que, en l'absence d'un examen sur l'objet lui-même, il reste difficile de réaliser un schéma très précis de ce processus de pliage.

[65] D'après les notes de fouilles de B. Bruyère, du mercredi 7 février 1951, archives IFAO ms_2004_0167_014 (https://www.ifao.egnet.net/bases/archives/bruye$\mathrm{re} /$ ?id=MS_2004_0167_014 dernière consultation 5/12/2019). Voir la citation dans KoeNIG 1999, p. 259.

[66] KoENIG 1999, p. 279. Sur ce motif, voir aussi les propositions d'interprétation de MORENZ 2013, p. 71-72 et ReEMES 2015, p. $194-195$ et p. 347, fig. 57.

[67] BRUYÈRE 1953, p. 114-115.
Les empreintes d'encre du PDM 44 ne doivent par ailleurs sans doute pas être considérées comme des indices de rapidité d'exécution de l'objet. II ne semble en effet guère probable que l'intégralité de l'encre (des 19 lignes de hiératique et de la vignette) ait encore été humide au moment du pliage, et il faut probablement envisager, sous toutes réserves, l'hypothèse que le papyrus, mis au jour au cours de la fouille du puits 1444, très perturbé [67], ait pu rencontrer, au cours de son histoire, un épisode humide qui aurait favorisé cette migration d'encre [68]. II ne s'agit là bien évidemment que de réflexions provisoires qui ont été faites à partir d'observation sur photographie et qu'il conviendrait d'approfondir.

\section{RECHARGES D'ENCRE ET PROCESSUS CRÉATIF}

La fréquence et la qualité des recharges d'encre constituent un indice matériel complémentaire qui, selon Chloé Ragazzoli [69], permet de déterminer le degré d'implication cognitive d'un scribe dans l'écriture de son texte. Comme cette chercheuse l'a récemment rappelé dans son étude sur les manuscrits des miscellanées ramessides, il existe deux types de recharges d'encre :

- l'une réalisée régulièrement afin que la densité de I'encre soit uniforme sur un manuscrit (calligraphic dipping), I'objectif étant de réaliser un beau document (par exemple, dans le cas du Livre des morts) ; - I'autre réalisée de façon plus irrégulière et systématiquement à un moment sémantiquement pertinent, au début d'une phrase ou d'un groupe nominal (meaningful dipping), avec, par conséquent, des densités d'encre très variables sur l'ensemble du manuscrit.

Ce dernier procédé de recharge suggérerait que le scribe est attentif au sens des mots qu'il est en train d'écrire. L'inventaire des recharges d'encre des papyrus-amulettes ramessides doit être réalisé sur un échantillon représentatif pour que des conclusions puissent être généralisées. À titre indicatif, on peut toutefois proposer un inventaire provisoire des recharges d'encre sur l'amulette PDM 36, dont une traduction [70] est donnée ci-dessous.

[68] Comparer avec BАВсоск \& EMMEL 1997, p. 240 (cité par CHANG 2014, p. 36-37)

[69] RAGAZZOLI 2017, p. 106-107

[70] Pour la traduction, voir SAUNERON 1970 (avec notes de traduction), ainsi que les corrections de lecture de QUACK 2011, p. 415, et le commentaire de BARDINET 1992 , p. 69 , n. 53, à propos de l'indication temporelle sur la manifestation de la maladie. Voir aussi DIELEMAN 2015, p. 27. 


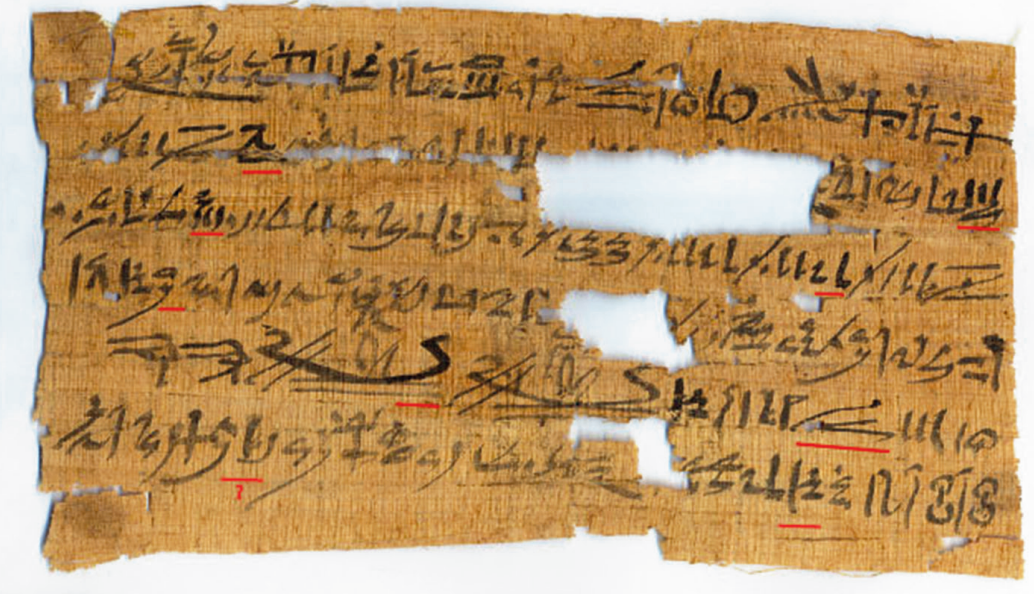

Figure 5

Recto du PDM 36 (@ IFAO - cliché nu_2013_00180), avec indication des recharges d'encre potentielles.
« Décret royal. C'est le roi de Haute et Basse Égypte Osiris qui dit au vizir et prince Geb : dresse ton mât (2), déploie (?) ta vo[ile, et pars pour les Cha]mps d'Ialou, en emmenant l'esprit possesseur nsy mâle et (3) l'esprit possesseur nsy femelle, I'opposant, l'opposante, le mort, la morte qui est face à Anynakht qu'a mis au monde Oubékhet (4), avec I'inflammation séref ( $p_{3}$ srf), la maladie réménet (t3 rmn.t), [et ...] mauvaise et néfaste, après qu'ils viennent contre lui sur (5) une période de trois jours (hr-tp hrw 3). Dire les paroles-divines SUr DEUX BARQUES, DEUX YEUX OUDJAT (6), DEUX SCARABÉES, tracés sur une feuille de papyrus neuve ( $\left.\underline{d} m^{\prime} n m_{3} w\right)$, à placer à son cou. Elle l'écartera (le mal) rapidement. »

L'inventaire des traces de recharge proposée ci-dessous est fait à partir d'une observation sur photographie numérique couleur (fig. 5).

Ligne 1. Densité de l'encre apparemment globalement plus uniforme que sur le reste du document. Ligne 2. Deux recharges : $1^{\circ}$ premier signe de la ligne 2 (premier signe du premier mot šzbw, ici « déploie (?) », $2^{\circ}$ groupe verbal in (impératif « emporte »).

Ligne 3. Deux recharges : $1^{\circ}$ début du mot $\underline{d}_{3 y}$ ( « adversaire »), $2^{\circ}$ signe $m s$ du mot $m s . n$ ( qu'a mis au monde »).

[71] Sur les parallèles textuels entre amulettes et recueils formulaires, voir KOENIG 2004, qui forge la notion « d'intertextualité sacrée » comme principe de création des textes des amulettes. Pour une recension des parallèles textuels des amulettes, voir FISCHER-ELFERT 2015, tableau, p. XVIII. Voir particulièrement la discussion autour des amulettes Berlin 15749 (utilisation de I'Hymne à l'uraeus) et P. hiérat. 3ab d'Heidelberg (hymne à l'uraeus combiné à une conjuration contre le démon Séhaqeq), FisCHER-ELFERT 2015, cat. 7 et 20, p. 230243. Sur I'amulette British Museum EA 10732, variante du billet « modèle »P. Chester Beatty VII verso 7, voir DONNAT, 2020, à paraître.
Ligne 4. Une seule recharge qui paraît certaine, en raison des lacunes, en fin de ligne : $r=f$ (« contre lui). Ligne 5. Trois recharges : $1^{\circ}$ le cadrat $\underline{d d}$ (《dire », début de la rubrique prescriptive), $2^{\circ}$ préposition $h r$ «sur » introduisant les images sur lesquelles prononcer les paroles, $3^{\circ}$ la deuxième image (le deuxième bateau).

Ligne 6. Pas de recharge nettement visible et encre assez claire sur la totalité de la ligne, une recharge vraisemblablement à la préposition $h r$ (《sur ») et peut-être, sous toutes réserves dans la séquence $r w i=f s w$ (« il/elle l'écartera ») et avec un degré d'incertitude pour le $r$ initial.

La densité de l'écriture, variable, et la qualité des recharges semblent indiquer qu'on est en présence d'un cas de meaningful dippings. Ce type d'observation (rapidité de l'écriture et nature des recharges d'encre) est bien sûr à associer à une analyse philologique des parallèles textuels et des variantes [71], permettant de tenter de comprendre, au cas par cas, le mode de composition des textes des amulettes : s'agit-il de (re)production à partir de la mémorisation, orale et visuelle, de textes de références [72] ? Les parallèles et variantes iconographiques [73] sont bien sûr à prendre aussi en compte pour déterminer le périmètre des savoirs religieux mobilisés pour la composition du dispositif graphique [74] dans son ensemble.

[72] Comparer avec la réflexion de AGUT-LABORDÈRE 2011 , p. 352-355, sur les modalités probables d'apprentissage des sagesses démotiques.

[73] FISCHER-ELFERT 2015, tableau p. XIX. Voir aussi, à titre d'exemple, la combinaison Thouéris-personnage anthropomorphe-crocodile (cf. supra, fig. 2) présente sur deux amulettes ramessides : KoENIG 1999, p. 276-279 ; REEMES 2015, p. $194-196$, p. 346-347 fig. 56-57 ; MORENZ 2013, p. 71-72.

[74] Sur la notion de dispositif graphique, CARASTRO 2009, p. 266-271. 


\section{UN POINT (RELATIVEMENT) AVEUGLE}

Si ces observations matérielles, combinées aux données textuelles, permettent de récolter des indices sur les modalités de composition, de fabrication et d'installation des amulettes, il demeure un point (relativement) aveugle dans ce dossier, déjà signalé en introduction : celui du destin final des amulettes, une fois que leur port n'était plus, pour diverses raisons, considéré comme nécessaire ou efficace. Cette question est, à ma connaissance, assez peu abordée dans les travaux égyptologiques [75]. La difficulté à prendre en considération cette question tient principalement à la nature et à l'état des sources. Les sources textuelles (essentiellement les notices prescriptives) insistent sur le moment d'activation de l'amulette (par l'énonciation de l'incantation) et sur le moment de son installation sur le corps de la personne à protéger. Elles sont en revanche totalement silencieuses sur l'ultime étape probable, celle de la "désinstallation " de l'amulette. En la matière, les provenances archéologiques pourraient être des données précieuses. Elles sont toutefois rarement connues, et, quand c'est le cas, les informations sont souvent peu précises. À Deir el-Médîna, on sait ainsi que l'amulette PDM 36 a été retrouvée en 1951 dans un kôm de déblais de fouilles, à l'est du Grand Puits [76] ; le PDM 44 provient quant à lui de la tombe P 1444 de Gournet Mourraï, mais est issu d'un contexte perturbé [77]. L'amulette MMA 26.3.225, datée de l'époque ramesside, a été trouvée dans la Tombe Thébaine 313, mais sans aucun autre vestige funéraire datable de la même époque. Les éditeurs du papyrus émettent ainsi l'hypothèse que l'amulette a été enterrée avec son possesseur, l'efficacité de l'amulette ayant ainsi, peut-être, été transposée dans le domaine funéraire. Elle serait l'unique artefact oublié lors de l'enlèvement du mobilier et de la momie au moment de la réutilisation de la tombe à la Basse Époque. D’autres publications de papyrus-amulettes, aussi découverts dans des contextes funéraires - à distinguer des amulettes réalisées pour des défunts (Totenbuchamulett) [78] -, sont à venir [79].

[75] Une exception notable : Dieleman \& FISCHERELFERT 2017, § 1.1, et note 6 avec références.

[76] SAUNERON 1970.

[77] Ms-2004-0167-014, cité plus haut, n. 65

[78] Supra, n. 10.

[79] Un papyrus-amulette, découvert dans la Tombe Thébaine 233 par l'équipe de la Macquarie University, est notamment en cours de publication par
En attendant, avec les précautions méthodologiques qui s'imposent, les parallèles ethnographiques [80] peuvent faire émerger des pistes de réflexion potentielles. L'Institut du Monde Arabe de Paris a ainsi accueilli, en 2013, une exposition sur les écrits talismaniques d'Afrique de l'Ouest. Le matériel exposé provenait d'objets et amulettes collectés dans la décharge à ordures de la ville de Dakar entre 1983 et 2012 [81]. Ce type de provenance pour des objets ethnographiques est propre à susciter la curiosité de l'archéologue. Le catalogue " enrichi » de l'exposition propose par ailleurs quelques réflexions sur les logiques qui peuvent pousser les personnes à déposer aux ordures un objet pourtant investi de puissance et portant des textes sacrés (coraniques) : "L'usage d'une amulette est personnel et sa durée de vie est variable. S'il lui est attribué une efficacité, elle sera précieusement conservée, transmise à des héritiers ou des amis, mais souvent disparaitra avec son possesseur. L'amulette peut être cassée ou gâtée du fait du contact avec des choses impures, désinvestie du fait de changement de croyances ou d'infortunes ", ce qui peut aboutir, commentent les auteurs, à " différentes circonstances de jetage ». Différents destins possibles pour les amulettes coraniques d'Afrique de l'Ouest sont ainsi inventoriés et commentés : transmission, enterrement, destruction, désinvestissement progressif, jetage selon diverses modalités [82]... Ces parallèles ethnographiques permettent d'envisager un panel de possibles pour interroger en retour les données égyptologiques. Dans l'attente d'un inventaire systématique des données archéologiques disponibles, on se gardera toutefois d'avancer des interprétations définitives pour le matériel pharaonique.

\section{CONCLUSION}

La prise en compte de la dimension matérielle des textes est bien évidemment un élément essentiel pour aborder un document écrit quel qu'il

H.-W. Fischer-Elfert, B. Ockinga et S. Binder. Voir le schéma et les informations techniques sur cette amulette dans KRUTZSCH 2015, p. 36.

[80] Pour un panorama comparatiste des amulettes écrites, voir CARDONA 1983.

[81] Epelboin, hamès, Laurent \& Durand 2013.

[82] Epelboin, hamès, Laurent \& Durand 2013, p. 12 et p. 14. 
soit [83]. Elle est particulièrement nécessaire dans le cas de l'étude d'objets inscrits pouvant être identifiés comme " écritures rituelles [84] ». L'approche matérielle du texte permet en effet d'observer les traces directes des gestes constitutifs d'une chaîne opératoire de fabrication. La comparaison (ethnographique), quant à elle, est une démarche opérée assez tôt en égyptologie pour ce type de matériel [85]. Méthodologiquement renouvelée par l'anthropologie historique contemporaine [86], l'approche comparative constitue un outil, à même de faire émerger des questionnements sur les manipulations mais aussi les circonstances énonciatives dans lesquelles un objet rituel peut être pris [87]. Les hypothèses formulées par ce biais doivent cependant être systématiquement évaluées à l'aune des données du terrain et de son contexte socio-historique.

Au terme de cet exposé, on posera une question, à laquelle il n'est pas aisé de répondre : l'ensemble des gestes, qui peuvent ainsi être reconstitués ou postulés par un raisonnement inductif ou analogique, peuvent-ils pour autant nécessairement être considérés comme « rituels [88] » ? C'est-à-dire, selon une définition usuelle du rite, peut-on considérer qu'ils $s^{\prime}$ inscrivaient dans un rapport particulier au temps, qu'ils étaient réalisés dans des conditions tout à fait spécifiques et non ordinaires ? Entretenaient-ils un lien particulier avec la parole énoncée ? Ce sont bien sûr les sources textuelles (internes et externes au corpus) qui constituent, en dernier ressort, le matériau privilégié sur lequel appuyer le travail interprétatif. Si on en croit les textes (les notices prescriptives), l'installation au cou de la personne à protéger était clairement ritualisée, puisqu'elle était accompagnée d'un acte verbal, l'énonciation de l'incantation. Quant aux conditions du tracé du texte et des dessins sur le papyrus [89], de plus amples recherches sont encore nécessaires : était-il réalisé en présence du commanditaire, ou plutôt préparé en amont de la consultation d'installation ? On peut supposer que le tracé du dispositif graphique devait se faire selon certaines modalités particulières, qui le distinguaient d'un acte d'écriture ordinaire. Dans les manuels de rituels, il existe des prescriptions stipulant l'usage d'encres spécifiques [90]. Toutefois, dans l'état actuel de la recherche, aucune ne me paraît pouvoir être très directement associée aux amulettes textuelles d'époque ramesside. En revanche, les notices prescriptives demandent régulièrement d'utiliser une feuille de papyrus vierge [91]. Cette prescription implique peut-être l'existence d'un cérémoniel minimal de l'écriture, au moins très modeste et intériorisé par le scribe. Elle signale en tout cas que cet acte d'écriture n'est pas un acte tout à fait ordinaire [92]. Elle est peut-être en cela à comparer aux conditions de pureté $\left(w^{\prime} b\right)$ qui sont requises pour la bouche de l'officiant [93] et qui contribuent à faire que l'énonciation rituelle n'est pas un acte de parole comme les autres. Il s'agit là sans doute d'une piste comparative qu'il convient d'approfondir pour en évaluer la pertinence et les implications potentielles sur le statut de l'écriture dans ce rite.

[83] Voir la « lecture » matérielle du restaurateur de papyrus, comparée à la lecture de l'égyptologue philologue : KRUTZSCH 2016, p. 58. Pour une approche matérielle de l'écrit, voir aussi de façon générale, EYRE 2018 et RAGAZZOLI 2019, p. 35-117.

[84] Supra, n 2.

[85] SAUnERON 1970. Cf. aussi les comparaisons sur le plan technique de KRUTZSCH 2015, p. 27-28 et fig. 11.

[86] CALAME 2012, p. 37.

[87] Voir par exemple, CARTRY 2009, sur la formulation orale qui accompagne la production graphique (fragment de calebasse couvert de signes) du devin gourmanché (population occupant la partie orientale du Burkina Faso) au cours d'une consultation géomantique.

[88] Voir le questionnement comparable de JAMBON 2010, à propos des inscriptions sur les figurines d'envoûtement et vases d'exécration pharaoniques.

[89] Cf. un questionnement similaire à propos de la temporalité du tracé des lettres aux morts : DoNNAT BEAUQUiER 2014, P. 138-143.

[90] Cf. la prescription d'un usage successif de I'orpiment et de la myrrhe pour le dessin de figures divines dans une amulette graphique sur lin du P. Leyde I 346 (Nouvel Empire, XVIII dynastie). BommAS 1999, p. 17 (III, 3-4) et p. 106-108. Voir aussi des prescriptions de dessins dans le corpus des Textes des sarcophages ou du Livre des morts, ESCHWEILER 1994, p. 101, p. 103105, p. 113-114. Sur l'encre ry.t w3d, voir QUACK 1998. Sur les recettes d'encres spéciales dans les papyrus magiques grecs, voir AufRÈRE 2001.

[91] Pour des contre-exemples, supra, n. 57 ; RÉGEN, à paraître.

[92] Voir encore, RÉGEN, à paraître, à propos de certaines pratiques spécifiques dans le traçage prescrites dans des notices de chapitres du Livre des morts.

[93] Papyrus de Turin CGT 54050 (= P. Turin cat. 1995 $+1996,2,1)$, ROCCATI 2010, p. 23 et 169. 
Agut-Labordère, Damien, 2011, Le sage et l'insensé. La composition et transmission des sagesses démotiques, Paris.

Agut-Labordère, Damien, \& Chauveau, Michel, 2011, Héros, magiciens et sages oubliés de l'Égypte ancienne. Une anthologie de la littérature en égyptien démotique, Paris.

Albert, Florence, \& GABOLde, Marc, 2013, «Le papyrus-Amulette de Lyon Musée des Beaux-Arts H 2425 », Égypte Nilotique et Méditerranée 6, 2013, p. 159-168.

AssmanN, Jan, 2000, Images et rites de la mort dans l'Égypte ancienne, Paris.

Azzam, Laila, 2017, « The Leiden Papyrus I $353 »$, Göttinger Mitzellen 253, p. 15-24.

Aufrère, Sydney Hervé, 2001, «Les encres magiques à composants végétaux contenant de l'armoise, de la myrrhe et divers ingrédients d'après les papyrus magiques grecs et démotiques », dans S. H. Aufrère (éd.), Encyclopédie religieuse de l'univers végétal II, Montpellier (Orientalia Monspeliensia XI), p. 374-376.

BAвCOCK, Robert G., \& EMMEL, Stephen, 1997, «A Mirror Text of Thucydides VII 33-35 », Archiv für Papyrusforschung 43, p. 239-245.

BARDINet, Thierry, 1992, «Des guérisons immédiates dans les papyrus médicaux de l'Égypte ancienne », dans D. Gourevitch (éd.), Maladie et maladies. Histoire et conceptualisation, Mélanges en I'honneur de M. Gmerk, Genève, p. 51-75.

BommAS, Martin, 1999, Die Mythisierung der Zeit. Die beiden Bücher über die altägyptischen Schalttage des magischen pLeiden I 346, Wiesbaden (Göttinger Orientforschungen IV Reihe Ägypten 37).

BRUYÈRE, Bernard, 1953, Rapport sur les fouilles de Deir El Médineh (années 1948 à 1951), Le Caire (Fouilles de I'Institut français d'archéologie orientale 26).

CALAME, Claude, 2012, «Comparatisme en histoire anthropologique des religions et regard transversal : le triangle comparatif », dans C. Calame, B. Lincoln (éd.), Comparer en histoire des religions antiques, Liège, p. 35-51.

Carastro, Marcello, 2009, « Les liens de l'écriture. Katadesmoi et instances de l'enchaînement », dans M. Cartry, J.L. Durand \& R. Koch Piettre (éd.), Architecturer l'invisible. Autels, ligatures, écritures, Turnhout (Bibliothèque de l'École des hautes études. Sciences religieuses 138), p. 263-291.

CARDONA, Giorgio R., 1981, Antropologia delle scritura, Torino.

CARDONA, Giorgio R., 1983, « Gli amuleti scritti: un excursus comparativo », La Ricerca Folklorica 8, p. 91-97.

Cartry, Michel, Durand, Jean-Louis, \& Koch-PietTre, Renée (éd.), 2009, Architecturer l'invisible. Autels, ligatures, écritures, Turnhout (Bibliothèque de l'École des hautes études. Sciences religieuses 138).

Chang, Ruey-Lin, 2014, Un dossier fiscal hermopolitain d'époque romaine, conservé à la Bibliothèque nationale et universitaire de Strasbourg, Le Caire (Publications de I'Institut Français d'Archéologie Orientale, Bibliothèque générale 46). Coulon, Laurent, 2010, « Ancienne Égypte. Les rites», dans G. Andreu-Lanoë (éd.), Inventaire de l'Égypte, Paris (coll. Inventaires de l'Encyclopaedia Universalis), p. 283-289.

Coulon, Laurent, 2018, «Review, H.-W. Fischer-Elfert, Magika hieratika in Berlin, Hannover, Heidelberg und München. $2015 \gg$, Orientalistische Literaturzeitung 113-2, p. 116-119.

Demichelis, Sara, 2000, «Le phylactère du scribe Boutehamon », Bulletin de I'Institut français d'archéologie orientale 100 , p. 267-273.

Dieleman, Jacco, 2015, «The materiality of textual amulets in Ancient Egypt», dans D. Boschung \& J. A. Bremmer (éd.) The Materiality of Magic, Paderborn (Morphomata 20), p. 23-58.

Dieleman, Jacco, \& Fischer-Elfert, Hans-Werner, 2017, « A Textual Amulet from Theban Tomb 313 (Papyrus MMA 26.3.225) », Journal of the American Research Center in Egypt 53, p. 243-257.

Donnat Beauquier, Sylvie, 2014, Écrire à ses morts. Enquête sur un usage rituel de l'écrit dans l'Égypte pharaonique, Grenoble.

Donnat, Sylvie, 2014a, « L'écrit comme trace de rituel en Égypte ancienne. L'exemple des lettres aux morts », Archimède [En ligne] 1, 2014, p. 88-95. Mis en ligne le 5-11-2014. URL : http://archimede.unistra.fr/publicationset-collections/revue-archimede/archimede-1-2014/archimede-1-2014-dossier-lecrit-comme-trace-de-rituel/

Donnat, Sylvie, 2014b, « Introduction. Pour une approche pluridisciplinaire du rituel », Archimède [En ligne] 1, 2014, p. 1-6. Mis en ligne le 5-11-2014. URL : http://archimede.unistra.fr/fileadmin/upload/DUN/archimede/Revue_ Archimede_RAHA/Numero_1/03_Archimede_N1_DONNAT_INT_Rituel.pdf

Donnat, Sylvie, 2015, «Écriture et rites dans l'Égypte pharaonique. Approche pragmatique de l'écrit cursif en contexte rituel », Annuaire EHESS. Comptes rendus des cours et conférences 2013-2014, p. 587-588.

Donnat, Sylvie, 2016a, «Les amulettes-médjat à l'époque ramesside », Bulletin de la Société française d'égyptologie 195-196, p. 36-46.

DONNAT, Sylvie, 2016b, « Un billet contre la chaleur-séref : le papyrus hiératique. 69 de la BNU de Strasbourg », Revue d'égyptologie 67, p. 1-30 et pl. I. 
Donnat, Sylvie, 2019a, « The concept of 'letter to the dead' and Egyptian funerary culture », dans R. Nyord (éd.), Concepts in Middle Kingdom Funerary Culture - Proceedings of the Lady Wallis Budge Anniversary Symposium held at Christ's College, Cambridge, 22 January 2016, Leiden - Boston (Mass.), p. 46-62.

DONNAT, Sylvie, 2019b, «Écriture rituelle», dans VOCES, Vocabulaire pour l'Étude des Scripturalités, Thomas Brunner (dir.), ARCHE EA3400(Université de Strasbourg), édition électronique (2015-), 2019-10-25 (mise en ligne), URL : http://num.ea3400.unistra.fr/voces/notice/ecriture_rituelle.xml.

DonnAT, Sylvie, 2020 à paraître, « Le papyrus-amulette British Museum EA 10732 et le billet "modèle" P. Chester Beatty VII verso 7 », Journal of Egyptian Archaeology 105, 2.

Epelboin, Alain, Hamès, Constant, LaRco-Laurent, Joana, \& DuRAnd, Jean-Louis, 2013, Un art secret. Écritures talismaniques d'Afrique de l'Ouest, Catalogue «enrichi de l'exposition (avril 2013) - Exposition du 14 février au 28 juillet 2013 à I'Institut du Monde Arabe, Paris. URL : https://issuu.com/institutdumondearabe/docs/art_secret_ catalogue_bd 1/06/2018.

EDWARDS, Iorwerth E.S., 1960, Hieratic Papyri in the British Museum. IVth Series Oracular Amuletic Decrees of the Late New Kingdom, London.

EDWARDS, Iorwerth E.S., 1968, « Kenḥikhopshef's prophylactic charm », Journal of Egyptian Archaeology 54, p. $155-160$.

EsChWeILeR, Peter, 1994, Bildzauber im alten Ägypten, Freiburg - Göttingen.

EYRE, Christopher, 2018, « The Material Authority of Written Texts in Ancient Egypt », dans P.A.J. Hoogendjick \& S. van Gompel (éd.), The Materiality of Texts from Ancient Egypt. New Approaches to the Study of Textual Material from Early Pharaonic to the Late Antique Period, Leiden - Boston, p. 1-11.

Fischer-Elfert, Hans-Werner, 1998, «Legenda Hieratika », Göttinger Miszellen 165, p. 105-112.

Fischer-Elfert, Hans-Werner, 2015, Magika hieratika in Berlin, Hannover, Heidelberg und München, Berlin.

FISCHER-ELFERT, Hans-Werner, à paraître, « Notizen zu eingen ägyptischen Wörten und Texten ».

FrankfURTeR, David, 2018, «'It is Esrmpe who appeals!' Place, Objects, and Performance in a Quest for Pregnancy in Roman Egypt », dans M. Ahuvia \& A. Kocar (éd.), Placing Ancient texts. The Ritual and Rhetorical Use of Space, Tübingen, p. 181-196.

GARDINeR, Alan H., 1915, « Magic (Egyptian) », dans Encyclopaedia of Religion and Ethics, by James Hasting, VIII, Edinburg, p. 262-269.

GARDINeR, Alan H., 1935, Hieratic Papyri in the British Museum, III ${ }^{r d}$ Series, London.

GermeUR, Ivan, 2013, «Entre magie et médecine : I'exemple du papyrus Brooklyn 47.218.2 », Égypte. Afrique \& Orient 71, p. 11-22.

GoodY, Jack, 1986, La logique de l'écriture. Aux origines des sociétés humaines, traduction de l'anglais, par AnneMarie Roussel, The Logic of Writing and the Organization of Society, Paris.

Goody, Jack, 2007, Pouvoirs et savoirs de l'écrit, traduction de I'anglais par Claire Maniez, coordination Jean-Marie Privat, Paris.

Grandet, Pierre, 2003, Catalogue des ostraca hiératiques non littéraires de Deir el-Médînéh, Le Caire (Documents de fouilles de I'IFAO 41).

Haro Sanchez, Magali, 2012, « Mise en contexte des papyrus iatromagiques grecs », dans P. Schubert (éd.), Actes du 26e congrès de papyrologie, Genève, p. 159-169.

ILLĖs, Orsolya, 2005, « Singe Spell Book of the Dead Papyri as Amulets », dans B. Backes, I. Munro \& S. Stöhr (éd.), Totenbuch-Forschungen: gesammelte Breiträge des 2. Internationalen Totenbuch-Symposiums, Bonn, 25. Bis 29. September 2005, Wiesbaden (Studien zum Altägyptischen Totenbuch 11), p. 121-150, pl. 1-3.

JAILlARD, Dominique, 2009, «Espaces hermaïques du sacrifice », dans M. Cartry, J.-L. Durand \& R. Koch Piettre (éd.), Architecturer l'invisible. Autels, ligatures, écritures, Turnhout (Bibliothèque de l'École des hautes études. Sciences religieuses 138), p. 61-79.

Jambon, Emmanuel, 2010, « Les mots et les gestes. Réflexions autour de la place de l'écriture dans un rituel d'envoûtement de l'Égypte pharaonique », Cahiers « Mondes anciens » [En ligne], 1 | 2010, 20 janvier 2010, consulté le 18 février 2019. URL : http://journals.openedition.org/mondesanciens mis en ligne le /158; DOI : 10.4000/ mondesanciens.158.

Koch-Piettre, Renée \& Batsch Jérôme, 2010, «Avant-propos», dans le dossier Écriture rituelle, Cahiers «Mondes anciens »[Online], 1 | 2010, mis en ligne le 19 January 2010, consulté le 18 février 2019. URL : http:// journals.openedition.org/mondesanciens/128.

Koenrg, Yvan, 1979, « Un revenant inconvenant ? (Papyrus Deir el-Médineh 37) », Bulletin de I'Institut français d'archéologie orientale au Caire 79, p. 103-119, pl. XXXVIII-XXXIX.

KoenIG, Yvan, 1981, « Les effrois de Keniherkhepeshef (Papyrus Deir el-Médineh 40) 》, Revue d'égyptologie 33, p. 33-37.

Koenrg, Yvan, 1999, « Le contre-envoûtement de Ta-i.di-Imen. Pap. Deir el-Médineh 44 », Bulletin de l'Institut français d'archéologie orientale au Caire 99, p. 259-281.

KoenIG, Yvan, 2004, «Le papyrus de Moutemheb », Bulletin de l'Institut français d'archéologie orientale au Caire 104, p. 291-326.

Koenrg, Yvan 2015, « Histoires sans paroles (P.Deir al-Medîna 45, 46, 47) », Bulletin de l'Institut français d'archéologie orientale au Caire 111, p. 243-256.

KRUTzSCH, Myriam, 2006, « Falttechniken an Handschriften aus dem alten Ägypten », dans B. Backes, I. Munro \& S. Stöhr (éd.), Totenbuch-Forschungen: gesammelte Breiträge des 2. Internationalen Totenbuch- Symposiums, Bonn, 25. bis 29. September 2005, Wiesbaden (Studien zum Altägyptischen Totenbuch 11), p. 167-195. 
KRUTzSCH, Myriam, 2015, « Materialtechnische Beobachtungen wähhrend der Restaurierung », dans Fischer-Elfert 2015, p. 1-74.

KRUTzSCH, Myriam, 2016, « Reading Papyrus as writing material », British Museum Studies in Ancient Egypt and Sudan 23, p. 57-69.

LefèVRE, Dominique, 2017, «Épistolographie et diplomatique : la rédaction d'une lettre aux dieux sous la XXIe dynastie », Classica et Semitica 10, p. 133-137.

LeITz, Christian, 1999, Hieratic Papyri in the British Museum VII series, London.

LUCARELLI, Rita, 2009, «Popular Beliefs in Demons in the Libyan Period: the Evidence of the Oracular Amuletic Decrees », dans G.P.F. Broekman, R.J. Demarée \& O.E. Kaper (éd.), The Libyan Period in Egypt. Historical and Cultural Studies into the 21th-24th Dynasties, Proceedings of a Conference at Leiden University 25-27 october 2007, Leiden, p. 231-239.

MathreU, Bernard, 2003, «La littérature égyptienne sous les Ramsès d'après les ostraca littéraires de Deir elMédineh », dans G. Andreu (dir.), Deir el-Médineh et la vallée des Rois : la vie en Égypte au temps des pharaons du Nouvel Empire. Actes du colloque organisé par le musée du Louvre, les 3 et 4 mai 2002, Paris, p. 117-132.

Morenz, Ludwig D., 2013, « Das Krokodil als göttliche Waffe in einer medico-magischen Bildkomposition aus Deir el Medineh », Archiv für Religiongeschichte 14, p. 69-82.

QUACK, Joachim F., 1998, « Mit grüner Tinte rot schreiben? », Göttinger Miszellen 165, p. 7-8.

QUACK, Joachim F., 2011, « Beiträge zu einigen Religiösen und Magischen Texten », dans M. Collier \& S. Snape (éd.), Ramesside Studies in Honour of K.A. Kitchen, Bolton, p. 413-416.

QUACK, Joachim F., 2014, « Die Drohung des Unlesbaren und die Macht des Ungelesenen », dans T. Frese, W.E. Kail \& K. Krüger (éd.), Verborgen, unsichtbar, unlesbar - zur Problematik restringierter Schriftpräsenz, Berlin, p. 32-42.

RAgAzzolI, Chloé, 2017, «Beyond authors and copyists. The role of variation in Ancient Egyptian and New Kingdom literary production », dans T. Gillen (éd.), (Re)productive Traditions in Ancient Egypt, Liège, p. 95-126.

RAgAzzolI, Chloé, 2019, Scribes. Les artisans du texte en Égypte ancienne, Paris.

RÉGEN, Isabelle, 2017, «The Archaeology of the Book of the Dead», dans F. Scalf (éd.), Book of the Dead. Becoming God in Ancient Egypt, Chicago (Oriental Institute Museum Publications 39), p. 97-108.

RÉGEN Isabelle, à paraître, «Entre rhétorique et pratique. L'archéologie du rituel dans le Livre des Morts », dans A. Pries \& F. Contardi (éd.), Tradition et transmission des rituels égyptiens : continuités et ruptures, Actes du colloque international, 17-19 mai 2017, Montpellier, Wiesbaden (Studien zur spätägyptischen Religion).

Reemes, Dana M., 2015, The Egyptian Ouroboros: An Iconological and Theological Study PhD University of California, Los Angeles, URL: http://escholarship.org/uc/item/6c0153p7 (dernière consultation août 2017).

Roccatr, Alessandro, 2011, Magica Taurinensia. Il libro magico di Torino e i suoi duplicati, Torino.

SAUNeron, Serge, 1970, « Le rhume d'Anynakhté (Pap. Deir el-Médinéh 36 », Kêmi 20, p. 7-18.

Sirat, Colette, 1990, «Introduction » dans C. Sirat, J. Irigoin \& E. Poulle (éd.), L'écriture : le cerveau, l'œil et la main, Turnhout, p. 3-19.

SKemer, Don C., 2014, « Magic Writ: Textuals Amulets Worn on the Body for Protection », dans A. Kehnel \& D. Panagiotopoulos (éd.), Schriftträger - Textträger: Zur materialen Präsenz des Geschriebenen in frühen Gesellschaften, Materiale Textkulturen 6, p. 127-150. En ligne : https://doi.org/10.1515/9783110371345.127 (dernière consultation 6/12/2019).

StAUder, Andréas, 2018, «Staging restricted Knowledge. The Sculptor Irtysen's self presentation (ca. 2000 BC) 》, dans G. Miniaci, J.C. Moreno García, St. Quirke \& A. Stauder (éd.), The Art of Making in Ancient Egypt. Voices, Images, and Objects of Material Producers (2000-1550 BC), Leiden, p. 239-271.

VAlbelle, Dominique \& Husson, Geneviève, 1998, « Les questions oraculaires d'Égypte : histoire de la recherche, nouveautés et perspectives », dans Egyptian Religion. The last thousand years, In Memoriam for Jan Quaegebeur, Leuven, p. 1055-1071.

Ventura, Jonathan, Popper-Giveon, Ariela \& Rabia, Atef abu, 2014 « Materialized 'Beliefs': Industrialized' Islamic Amulets $\gg$, Visual ethnography 3/2, janvier, p. 30-47.

Vernus, Pascal, 2010-2011, "Littérature", "littéraire" et supports d'écriture. Contribution à une théorie de la littérature dans l'Egypte pharaonique », Egyptian and Egyptological Documents, Archives, Libraries II, p. 19-145.

WeIss, Lara, 2014, «The Power of the Voice», dans B. Haring, O. Kaper \& R. van Walsem (éd.), Studies in Hieratic and the Documents of Der el-Medina, Egyptologische Uitgaven 28, Leiden, p. 291-303.

WeNDRICH, Willeke, 2006, «Entangled, connected or protected. The power of knots and knotting in ancient Egypt », dans K. Szpakowska (éd.), Through a Glass Darkly. Magic, dreams and prophecy in ancient Egypt, Oxford, p. 243-269. WeNTE, E.F., 1990, Letters from Ancient Egypt, Atlanta.

WINAND, Jean, 2003, «Les décrets oraculaires pris en I'honneur d'Henouttaouy et de Maâtkarê (Xe et VII pylônes) 》, Cahiers de Karnark 11, p. 603-705, pl. I-III.

YAMAZAKI, Naoko, 2003, Zaubersprüche für Mutter und Kind: Papyrus Berlin 3027, Berlin. 